\title{
Analog Circuits With High-Gain Topologies Using a-GIZO TFTs on Glass
}

\author{
Pydi Ganga Bahubalindruni, Bruno Silva, Vítor Grade Tavares, Pedro Barquinha, Nuno Cardoso, \\ Pedro Guedes de Oliveira, Rodrigo Martins, and Elvira Fortunato
}

\begin{abstract}
This paper presents analog building blocks that find potential applications in display panels. A buffer (source-follower), subtractor, adder, and high-gain amplifier, employing only n-type enhancement amorphous gallium-indium-zinc-oxide thin-film transistors (a-GIZO TFTs), were designed, simulated, fabricated, and characterized. Circuit simulations were carried out using a neural model developed in-house from the measured characteristics of the transistors. The adder-subtractor circuit presents a power consumption of $0.26 \mathrm{~mW}$, and the amplifier presents a gain of $34 \mathrm{~dB}$ and a power consumption of $0.576 \mathrm{~mW}$, with a load of $10 \mathrm{M} \Omega / / 16 \mathrm{pF}$. To the authors' knowledge, this is the highest gain reported so far for a single-stage amplifier with a-GIZO TFT technology.
\end{abstract}

Index Terms-Analog circuits, a-GIZO TFT, neural modeling.

\section{INTRODUCTION}

$\mathbf{L}$ OW-TEMPERATURE fabrication of amorphous Gallium-Indium-Zinc-Oxide (a-GIZO) based thin-film-transistors (TFT) [1], [2] is proving to be promising in display applications. Compared to other competing TFT technologies, such as OTFT [3] and a-Si:H [4], a-GIZO TFTs show superior electrical mobility [5] and reliability [6]. Even though it was mainly meant for backplanes of AMOLED [7] and ultra definition LCDs [8], it is also finding extensive applications in driving circuits for back-planes of displays [9], [10] and RFIDs [11]. However, a-GIZO TFTs imposes various challenges in circuit design. Lack of commercial device models and technology libraries makes the design and simulation complex. In addition, poor intrinsic mobility compared to crystalline silicon and the absence of stable complementary devices (p-type TFT), makes the design of high-gain amplifier stages more challenging.

Manuscript received October 16, 2014; accepted December 01, 2014. Date of publication December 04, 2014; date of current version May 13, 2015. This work was supported by the ERDF through the Programme COMPETE and by the Portuguese Government through FCT-Foundation for Science and Technology under Project CMU-PT/SIA/0005/2009, (FCOMP-01-0124FEDER-013070), strategic Project PEST-C/CTM/LA0025/2013-14, and by the European Research Council through the Advanced Grant INVISIBLE under Grant ERC-2008-AdG 228144. The work of P. G. Bahubalindruni was supported in part by the FCT under Grants BD/62678/2009.

P. G. Bahubalindruni, B. Silva, V. G. Tavares, N. Cardoso, and P. G. de Oliveira are with INESC TEC (formerly INESC Porto) and Faculty of Engineering, University of Porto, 4200-465 Porto, Portugal (e-mail: vgt@fe.up.pt).

P. Barquinha, R. Martins, and E. Fortunato are with CENIMAT/I3N, Departamento de Ciência dos Materiais, Faculdade de Ciências e Tecnologia, FCT, Universidade Nova de Lisboa and CEMOP-UNINOVA, 2829-516 Caparica, Portugal.

Color versions of one or more of the figures in this paper are available online at http://ieeexplore.iee.org.

Digital Object Identifier 10.1109/JDT.2014.2378058
Nevertheless, an operational amplifier was already reported with a gain of $18.7 \mathrm{~dB}$ [12] using enhancement TFTs, in which the amplifiers' first stage gain is limited due to the conventional diode connected loads. However, from a more practical point of view, in most cases this gain is not sufficient, demanding for more or higher gain stages.

The a-GIZO technology is fairly recent and has not yet reached the level of maturity that other technologies show. In general, reports on complex circuits is scarce, without topologies that favors the desired high gains. But in general, designing amplifiers with amorphous oxide-devices is a challenge because the gain is majorly impaired by the absence of stable complementary devices. Other techniques need to be employed to increase the active loads. In fact, one possibility is to use bootstrapping techniques to realize high active impedances. This type of technique was adopted in the past in bipolar technology to attain high-input impedances [13]. However, instead of the input, this very same technique can be used to boost the gain by applying the same principle to the amplifiers load. Such a concept can effectively be applied to single stage amplifiers to enhance the gain, up to values of the order of magnitude of the transistor intrinsic gain, and forms the bases for the proposed double bootstrapping technology. Then, this paper focuses on the design of analog blocks [14], [15] that are useful for high-gain stages, which can also function as buffer, adder and subtractor. All these functional blocks are important for data driver circuits in TFT LCD panels. In order to perform circuit simulations, a neural model was developed from measured characteristics of the transistors.

The rest of the paper is organized as follows. Section II presents the device characterization and modeling. Section III elaborates on the design of basic analog circuits and its operation (buffer, subtractor, and adder), including the proposed high-gain amplifier. Section IV presents the simulation results confronted with measured responses and finally in Section V conclusions are drawn.

\section{TFT CHARACTERIZATION AND MODELING}

A bottom-gate staggered structure for TFTs was employed in all circuits. This structure is widely adopted by the display industry due to its good process compatibility. The a-GIZO devices were fabricated by sputtering, with maximum processing temperature of $200^{\circ} \mathrm{C}$. All the fabrication details are identical to the ones in [16], except for source-drain electrodes, where in the present work e-beam evaporated $\mathrm{Ti} / \mathrm{Au}$ is used to improve the device performance and enable easier wire-bounding. The device modeling method follows a black-box approach and uses 


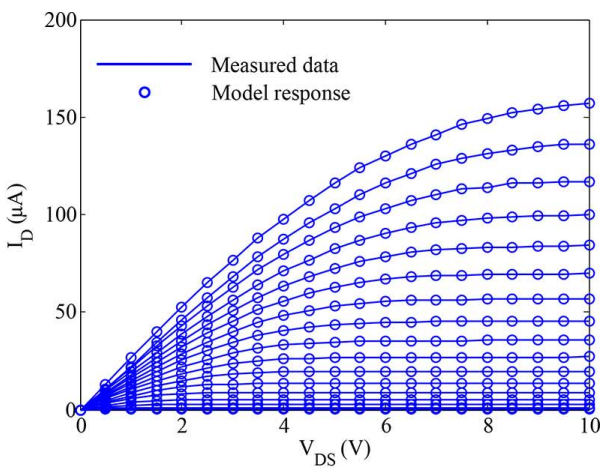

(a)

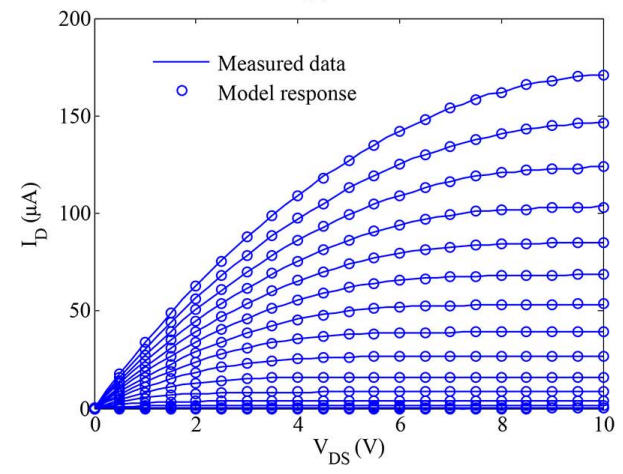

(b)

Fig. 1. Output characteristics of a-GIZO TFTs $(\mathrm{W} / \mathrm{L}=160 / 20 \mu \mathrm{m})$ with different layouts: $0 \leq \mathrm{V}_{\mathrm{DS}} \leq 10 \mathrm{~V}$ and $0 \leq \mathrm{V}_{\mathrm{GS}} \leq 8 \mathrm{~V}$, in steps of $0.5 \mathrm{~V}$. (a) Direct layout. (b) Fingered layout.

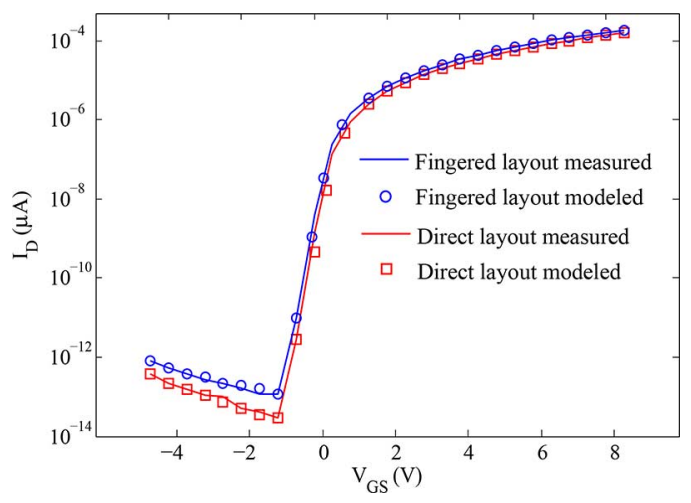

Fig. 2. Transfer characteristics of a-GIZO TFTs $(\mathrm{W} / \mathrm{L}=160 / 20 \mu \mathrm{m})$ with direct and fingered layouts, $\mathrm{V}_{\mathrm{DS}}=10 \mathrm{~V}$ and $-5 \leq \mathrm{V}_{\mathrm{GS}} \leq 8 \mathrm{~V}$ in steps of $0.5 \mathrm{~V}$.

artificial neural networks, as functional approximators, developed from measured characteristics of the transistors [14], [16].

It is recognized that the series contact resistance $\left(R_{S D}\right)$ at the source and drain terminals of TFTs lowers the performance of the device. $\mathrm{R}_{\mathrm{SD}}$ includes the contact resistance between the source/drain electrode to the semiconductor layer and the highimpedance path in the semiconductor between the source/drain and the conductive channel. Part of the applied bias voltages are dropped in the series resistance of the transistor resulting in less drain current, which in turn lowers the device mobility and shows strong impact on the circuit performance. In order to attenuate this problem, when the transistor width is greater than $40 \mu \mathrm{m}$, fingered layout is employed to minimize $\mathrm{R}_{\mathrm{SD}}$ on

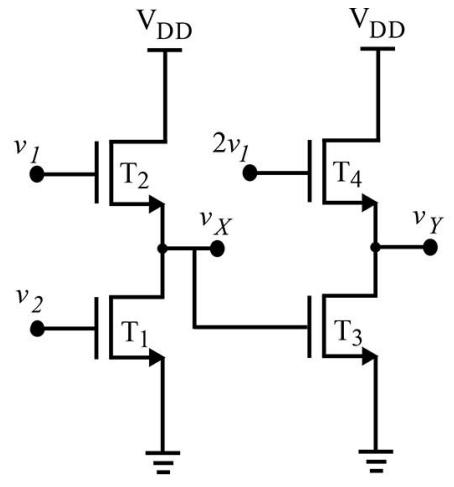

(a)

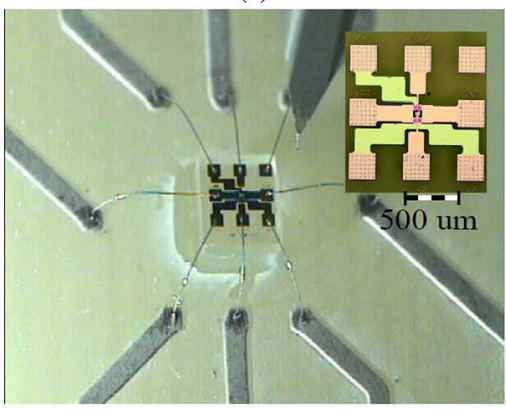

(b)

Fig. 3. Adder subtractor circuit. (a) Schematic. (b) Fabricated chip after wirebonding and the circuit micrograph in the inset, total circuit area (including pads) is $1250 \times 1250 \mu \mathrm{m}^{2}$.

each transistor, which will result on a bigger effective $V_{G S}$ and $\mathrm{V}_{\mathrm{DS}}$ (transistors are in parallel) for the same external bias, when compared to the single full width transistor. Under the same conditions, fingered layout shows higher drain current due to the higher effective $\mathrm{V}_{\mathrm{GS}}$ (can be seen as lower $\mathrm{V}_{\mathrm{TH}}$ ), and the lower total resistance also results in increased mobility, thereby improving the overall performance of the transistor.

The TFT output and transfer characteristics (measured and Verilog-A neural model response) are shown in Figs. 1 and 2, respectively, with both layouts. As it can be noticed, TFT with fingered layout presents more drain current than the direct layout transistor, for the same bias voltages and aspect ratio, which is explained by the effective lower series resistances in the fingered layout compared to the direct layout, as referred above.

\section{Circuit Design}

The adder-subtractor circuit schematic and its fabricated chip after wire-bonding are presented in Fig. 3(a) and (b), respectively. Based on the type of the inputs, it can function as a buffer, subtractor or adder. In addition, this block can be used to provide positive feedback (FB) for high-gain amplifier designs [17]-[19], as shown in Fig. 4. This circuit forms the basis for the different basic cells presented bellow.

\section{A. Source Follower}

A common-drain amplifier is useful in either shifting the DC level of a signal or as a buffer to drive circuits that present a low input impedance. In Fig. 3(a) the buffer circuit is formed with $\mathrm{T}_{1}$ and $\mathrm{T}_{2}$, by applying a constant bias $\left(\mathrm{V}_{2}\right)$ to $\mathrm{T}_{1}$ and the input signal $\left(v_{1}\right)$ to $\mathrm{T}_{2}$. 


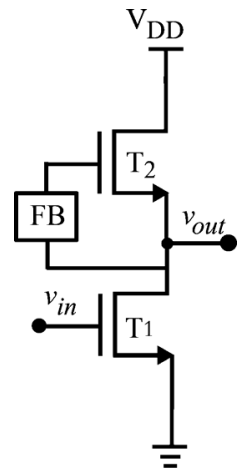

Fig. 4. High-gain amplifier topology using enhancement mode n-type transistors.

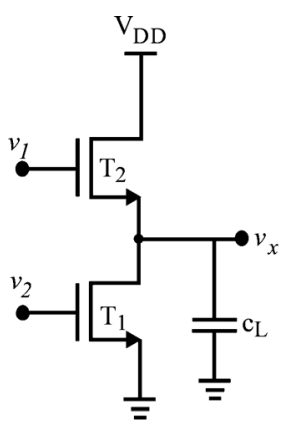

(a)

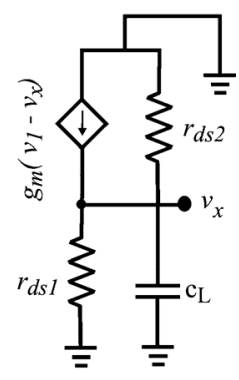

(b)
Fig. 5. Source follower configuration (a) Circuit schematic (b) Small signal equivalent circuit.

The schematic and the small signal equivalent circuit of the resulting source follower is shown in Fig. 5. The output $\left(v_{x}\right)$, follows the input $\left(v_{1}\right)$. The relation between $v_{x}$ and $v_{1}$ is given by,

$$
\frac{v_{x}}{v_{1}}=\frac{g_{m 2}}{g_{m 2}+g_{d s 2}+g_{d s 1}},
$$

where $g_{m}$ and $g_{d s}\left(=\frac{1}{r_{d s}}\right)$ are the transconductance and output conductance of the transistor. Unlike CMOS transistors, TFTs do not show body-effect, since the bulk is a pure insulator. Hence, a gain very close to unity can be obtained, when $g_{m} \gg$ $g_{d s}$.

\section{B. Adder-Subtractor}

Analog subtractor and adder circuits are important functional blocks in computational systems. In Fig. 3(a), $\mathrm{T}_{1}$ and $\mathrm{T}_{2}$ TFTs form the subtractor, whereas $\mathrm{T}_{1}-\mathrm{T}_{4}$ together constitutes the adder. Assuming all transistors in saturation, and that the drain current $\left(i_{\mathrm{D}}\right)$ can be approximately expressed by

$$
i_{D} \approx K *\left(v_{G S}-V_{T H}\right)^{2}
$$

it can easily be demonstrated that when all transistors operate in the saturation region, subtraction and addition of the input signals can be obtained at the following nodes:

$$
\begin{aligned}
& v_{X}=v_{1}-v_{2}, \\
& v_{Y}=v_{1}+v_{2} .
\end{aligned}
$$

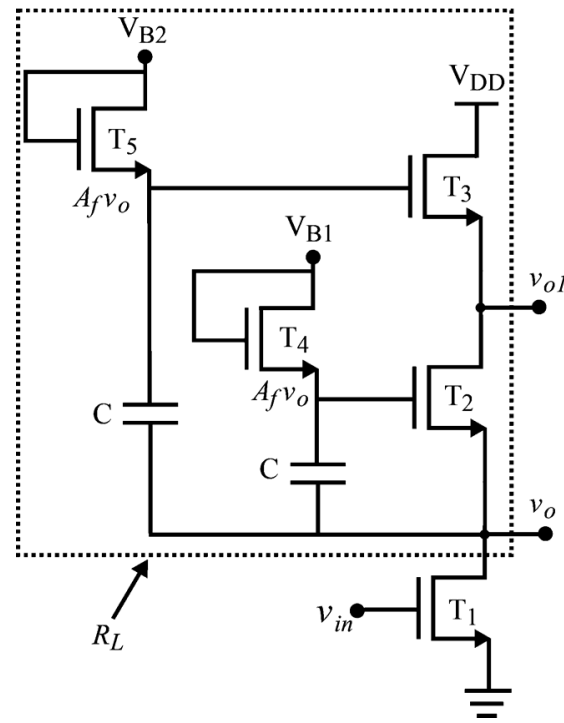

Fig. 6. Proposed high gain amplifier only with n-type enhancement mode transistors $\mathrm{P}$.

\section{Amplifier}

Fig. 6 presents a circuit proposed to enhance the gain of a single-stage common source amplifier, involving only n-type transistors (enhancement). It employs a double bootstrapping technique that increases significantly the equivalent active-load value, to a point where the global gain gets very close to the intrinsic gain of the input transistor. Fig. 7 depicts the fabricated circuit (hereafter referred to as "Amp") in two versions, one with on-chip capacitors and another with external.

Operation: In order to achieve amplification, transistors $\mathrm{T}_{1}, \mathrm{~T}_{2}$ and $\mathrm{T}_{3}$ should operate in saturation. The bias transistors $\mathrm{T}_{4}$ and $\mathrm{T}_{5}$ operate in cutoff during the DC steady state, because they come in series with a capacitor and high impedance branches from $\mathrm{T}_{2}$ or $\mathrm{T}_{3}$ gates (ideally the gate current is zero). Therefore, the biasing transistors represent high equivalent resistors for signal. Accordingly, the bias voltages $\mathrm{V}_{\mathrm{B} 1}$ and $\mathrm{V}_{\mathrm{B} 2}$ appear at the gate terminals of the transistors $\mathrm{T}_{2}$ and $\mathrm{T}_{3}$, respectively. By choosing proper values for the biasing voltages and power supply, operation in saturation can then be ensured for transistors $\mathrm{T}_{1}, \mathrm{~T}_{2}$ and $\mathrm{T}_{3}$. The output signal $\left(v_{o}\right)$ is fed back to transistors $\mathrm{T}_{2}$ and $\mathrm{T}_{3}$ by means of the feedback circuit that is formed by the capacitor $\mathrm{C}$ and the bias transistors $\left(\mathrm{C}, \mathrm{T}_{4}\right)$ and $\left(\mathrm{C}, \mathrm{T}_{5}\right)$, respectively. In small-signal terms, the biasing transistors equivalent circuit can then be represented by a parallel combination of the off-resistance $\left(R_{\mathrm{OFF}}\right)$ and parasitic capacitance $\left(\mathrm{C}_{\mathrm{OFF}}\right)$, as shown in Fig. 8(a) and (b). The load resistance $\left(R_{L}\right)$, from Fig. 8(a) is given by

$$
R_{L}=\frac{v_{o}}{i_{o}}
$$

Before finding the actual value of the load resistor, it should be noted that the feedback paths in the "Amp" circuit cause a high-pass filter action. Consequently, this amplifier cannot amplify DC. However, the lower cutoff frequency can be made very small ( $\mathrm{mHz}$ range), by choosing a proper high value for C. From Fig. $8(\mathrm{~b})$, the feedback gain $\left(A_{f}\right)$ is given by 


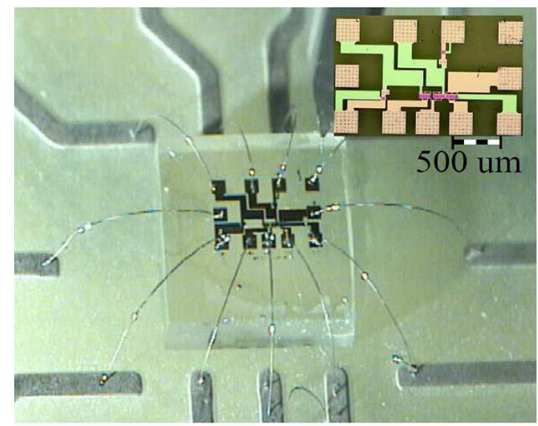

(a)

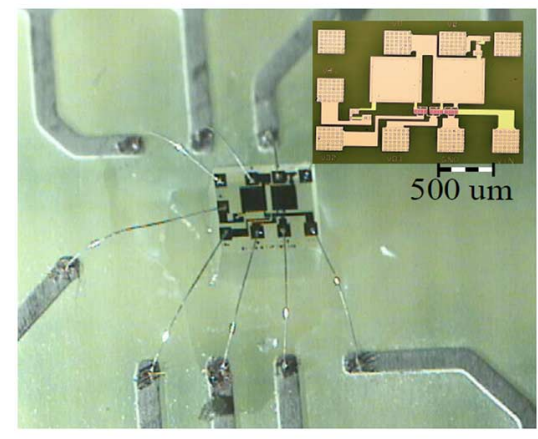

(b)

Fig. 7. Proposed bootstrap amplifier fabricated circuits after wire-bonding and the corresponding micrographs in the inset. (a) External capacitance. (b) On-chip capacitance.
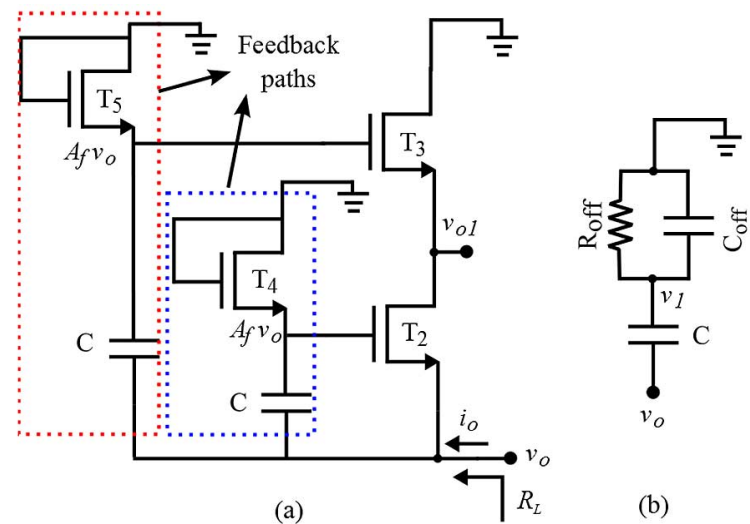

(b)

Fig. 8. Active load (a) Schematic (b) Feedback path (C, T4) or (C,T5).

$$
A_{f}=\frac{v_{1}}{v_{0}}
$$

where

$$
v_{1}=v_{0} \frac{\left(R_{O F F} / / \frac{1}{s C_{O F F}}\right)}{\frac{1}{s C}+\left(R_{O F F} / / \frac{1}{s C_{O F F}}\right)}
$$

since $\mathrm{R}_{\mathrm{OFF}}$ is very high, it can be simplified to

$$
\begin{aligned}
& v_{1}=v_{0} \frac{1}{1+\frac{C_{O F F}}{C}} \\
& A_{f}=\frac{v_{1}}{v_{0}}=\frac{1}{1+\frac{C_{O F F}}{C}} .
\end{aligned}
$$

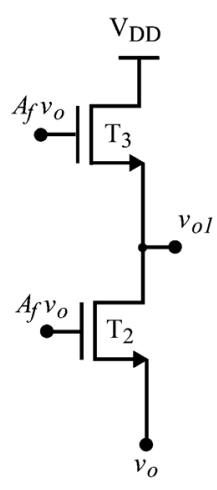

(a)

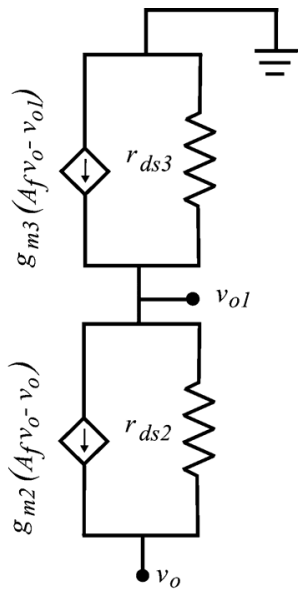

(b)
Fig. 9. (a) Simplified load (b) Corresponding small signal equivalent circuit.

Consequently, the amplifier with the simplified load can now be redrawn in the form shown in Fig. 9. Assuming all transistors with the same small-signal parameters, $A_{f} \approx 1$ and that $g_{m} \gg$ $g_{d s}$,

$$
\begin{aligned}
i_{o} & \approx \frac{g_{d s}^{2}}{g_{m}} v_{o} \\
R_{L} & =\frac{v_{o}}{i_{o}} \approx g_{m} r_{d s}^{2}
\end{aligned}
$$

The "Amp" gain is then given by

$$
\frac{v_{0}}{v_{i n}}=-g_{m}\left(r_{d s} / / g_{m} r_{d s}^{2}\right)
$$

which can basically be reduced to the intrinsic gain of the input transistor. This gain could be increased even further by employing a cascode driver.

\section{Results AND Discussion}

In order to simulate the above circuits, the developed Verilog-A neural model is used in Cadence Virtuoso Spectre simulator. Since the TFT intrinsic capacitance is not characterized, approximate values of bias dependent capacitance ( $\mathrm{c}_{\mathrm{gd}}$ and $\mathrm{c}_{\mathrm{gs}}$ ) are calculated from the Meyer's capacitance model for FETs, with a relative dielectric constant $\left(\epsilon_{r}\right)$ of 10.5 for the dielectric material. In addition, the load impedance offered by the measuring cable $(10 \mathrm{M} \Omega / / 16 \mathrm{pF})$ is also considered for all circuits. The power supply is $20 \mathrm{~V}$.

\section{A. Buffer}

Simulation results with the measured circuit response are shown in Fig. 10. Under the load conditions described above, this circuit presents a bandwidth of $40 \mathrm{kHz}$.

\section{B. Adder-Subtractor}

Functional verification as subtractor and adder is carried out for the following stimulus,

$$
\begin{aligned}
& v 1=9+\sin \left(2 \pi f_{1} t\right), \\
& v 2=4.5+0.5 * \sin \left(2 \pi f_{2} t\right) .
\end{aligned}
$$




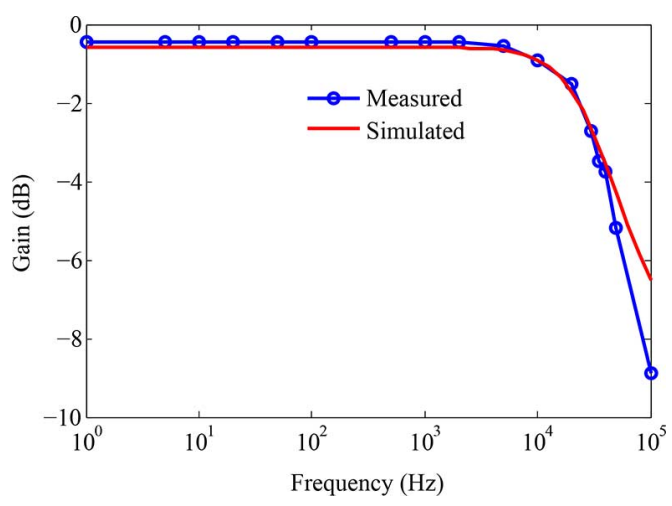

Fig. 10. Source follower simulation and measured circuit response.

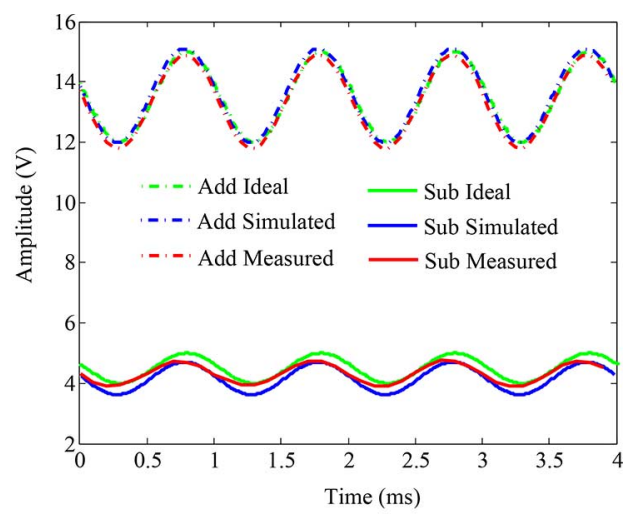

(a)

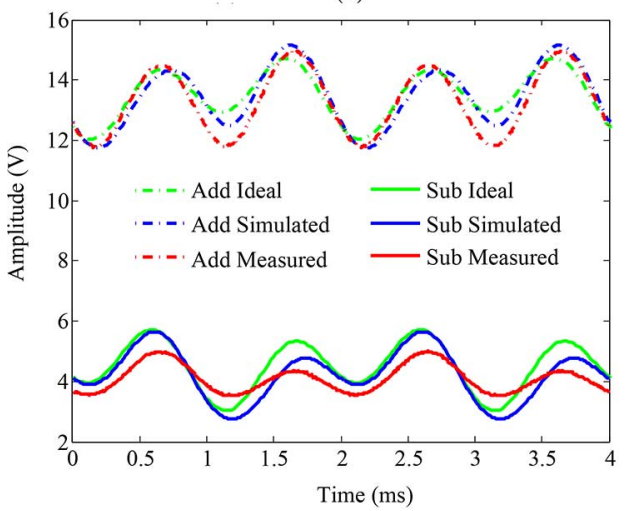

(b)

Fig. 11. Adder-subtractor functional verification from simulation, measurements, and expected value. (a) Same frequency. (b) Different frequency.

Verification is performed with two input signals $\left(v_{1}\right.$ and $\left.v_{2}\right)$ with the same frequency $\mathrm{f}_{1}=\mathrm{f}_{2}=1 \mathrm{kHz}$ and also with different frequencies $\mathrm{f}_{1}=1 \mathrm{kHz}$ and $\mathrm{f}_{2}=500 \mathrm{~Hz}$. Since the ANN verilog-A model cannot characterize the bias stress effect, a minor mismatch between the simulated and measured circuit response can be observed in Fig. 11. Non-idealities of the devices such as output resistance $\left(\mathrm{r}_{d s}\right)$ and minor mismatches between them are the other possible causes for the deviation observed between the ideal and the measured/simulated response. However, all outcomes show the same trend, which also demonstrates that the model is able to capture the device behavior.

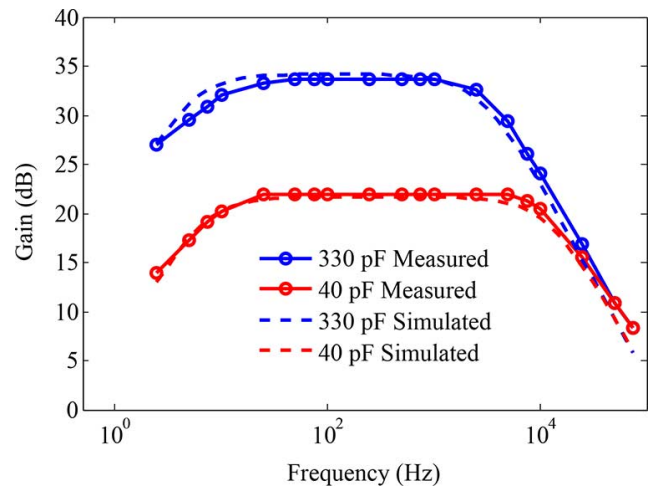

Fig. 12. Gain variation with respect to external and on-chip capacitance in high-gain amplifier. Dashed lines represent simulated data, solid lines with circles represent measured response.

TABLE I

Proposed High-Gain AMPLIFIER PERformance COMPARISON With LITERATURE

\begin{tabular}{|l|l|l|l|l|l|l|}
\hline Circuit & $\begin{array}{l}\text { No. } \\
\text { of } \\
\text { TFTs }\end{array}$ & $\begin{array}{l}\mathrm{C} \\
(\mathrm{pF})\end{array}$ & $\begin{array}{l}\text { Power } \\
(\mathrm{mW})\end{array}$ & $\begin{array}{l}\text { Bandwidth } \\
(\mathrm{kHz})\end{array}$ & $\begin{array}{l}\text { Mid- } \\
\text { band } \\
\text { gain } \\
(\mathrm{dB})\end{array}$ & Load \\
\hline $\mathrm{Amp}$ & 5 & 40 & 0.576 & 20 & 22 & $10 \mathrm{M} \Omega / / 16 \mathrm{pF}$ \\
\hline $\mathrm{Amp}$ & 5 & 330 & 0.576 & 5 & 34 & $10 \mathrm{M} \Omega / / 16 \mathrm{pF}$ \\
\hline$[12]$ & 16 & - & 0.9 & 54 & 18.7 & $1 \mathrm{M} \Omega / / 2 \mathrm{pF}$ \\
\hline
\end{tabular}

\section{High-Gain Amplifier (Amp)}

The "Amp" gain variation with respect to $\mathrm{C}$ is presented in Fig. 12. For high $\mathrm{C}$ values $(330 \mathrm{pF})$, external capacitors are utilized together with the circuit in the chip presented in Fig. 7(a). The second circuit uses on-chip capacitors with smaller values $(40 \mathrm{pF})$ as presented in Fig. 7(b).

Transistors $T_{1}-T_{3}$ were designed with the same aspect ratios $(\mathrm{W}=160 \mu \mathrm{m}$ and $\mathrm{L}=20 \mu \mathrm{m})$ with fingered layout, while, $\mathrm{T}_{4}$ and $\mathrm{T}_{5}$ were set with $\mathrm{W}=10 \mu \mathrm{m}$ and $\mathrm{L}=20 \mu \mathrm{m}$ in a direct-layout structure. Fig. 12 presents the Amp frequency response with different values of capacitance $(\mathrm{C})$, including a load (from the measuring cables) similar to the above mentioned circuits. From Fig. 12, it can be noticed that a higher value of $\mathrm{C}$ leads to an improvement in the gain, since the feedback factor becomes closer to unity, as shown in (7). A comparison of circuit complexity, power consumption, bandwidth and gain is presented in Table I. It can be noticed that a significant improvement in the gain and reduction in the power consumption is accomplished with the proposed capacitive bootstrapping technique.

\section{CONCLUSION}

Various analog circuit blocks, such as a buffer, adder-subtractor, and a high-gain amplifier were successfully designed and characterized using a-GIZO TFT. The simulation results, accomplished with the developed neural models, were in agreements with the measured response, showing the effectiveness of the modeling procedure. A new double-bootstrap amplifier was proposed for gain improvement. To the authors' knowledge, it presents the highest gain reported so far from a single-stage 
topology, with a-GIZO TFT technology. This gain could be increased further by augmenting the total active load with a cascode driver, instead of the single common-source transistor.
[19] P. Bahubalindruni, V. G. Tavares, P. Barquinha, R. Martins, and E. Fortunato, "High-gain topologies for transparent electronics," in Proc. IEEE EUROCON, Jul. 2013, pp. 2041-2046.

\section{ACKNOWLEDGMENT}

The authors would like to thank Dr. J. Borme from INL-Braga for the chip dicing and wire-bonding.

\section{REFERENCES}

[1] K. Nomura, H. Ohta, A. Takagi, T. Kamiya, M. Hirano, and H. Hosono, "Room-temperature fabrication of transparent flexible thin-film transistors using amorphous oxide semiconductors," Nature, vol. 432, no. 7016, pp. 488-492, 2004.

[2] E. Fortunato, P. Barquinha, and R. Martins, "Oxide semiconductor thin-film transistors: A review of recent advances," Adv. Mater., vol. 24, no. 22, pp. 2945-2986, 2012.

[3] H. Marien, M. Steyaert, E. van Veenendaal, and P. Heremans, "A fully integrated $\triangle \Sigma$ ADC in organic thin-film transistor technology on flexible plastic foil," IEEE J. Solid-State Circuits, vol. 46, no. 1, pp. 276-284, Jan. 2011.

[4] A. Dey and D. Allee, "Amorphous silicon 5 bit flash analog to digital converter," in Proc. Custom Integr. Circuits Conf., 2012, pp. 1-4.

[5] P. Barquinha, L. Pereira, G. Gonçalves, R. Martins, and E. Fortunato, "Toward high-performance amorphous GIZO TFTs," J. Electrochem. Soc., vol. 156, no. 3, pp. H161-H168, 2009.

[6] M. Lopes et al., "Gate-bias stress in amorphous oxide semiconductors thin-film transistors," Appl. Phys. Lett., vol. 95, no. 6, 2009, Art. ID 063502.

[7] J. Y. Kwon et al., "Bottom-gate gallium indium zinc oxide thin-film transistor array for high-resolution AMOLED display," IEEE Electron Device Lett., vol. 29, no. 12, pp. 1309-1311, Dec. 2008.

[8] N. Gong et al., "58.2: Distinguished paper: Implementation of $240 \mathrm{hz}$ 55-inch ultra definition LCD driven by a-IGZO semiconductor TFT with copper signal lines," in SID Symp. Dig. Tech. Papers, 2012, vol. 43, no. 1, pp. 784-787.

[9] B. Kim et al., "A depletion-mode In-Ga-Zn-O thin-film transistor shift register embedded with a full-swing level shifter," IEEE Electron Device Lett., vol. 58, no. 9, pp. 3012-3017, Sept. 2011.

[10] B. Kim et al., "A depletion-mode a-IGZO TFT shift register with a single low-voltage-level power signal," IEEE Electron Device Lett., vol. 32, no. 8, pp. 1092-1094, Aug. 2011.

[11] H. Ozaki, T. Kawamura, H. Wakana, T. Yamazoe, and H. Uchiyama, "20-uw operation of an a-IGZO TFT-based RFID chip using purely NMOS "active"; Load logic gates with ultra-low-consumption power," in Proc. Symp. VLSI Circuits, Jun. 2011, pp. 54-55.

[12] C. Zysset, N. Munzenrieder, L. Petti, L. Buthe, G. Salvatore, and G. Troster, "Igzo TFT-based all-enhancement operational amplifier bent to a radius of $5 \mathrm{~mm}$," IEEE Electron Device Lett., vol. 34, no. 11, pp. 1394-1396, Nov. 2013.

[13] T. D. Towers, "High input-impedance amplifier circuits," Wireless World, pp. 197-201, July 1968.

[14] G. Bahubalindruni et al., "Basic analog circuits with a-GIZO thin-film transistors: Modeling and simulation," in Proc. IEEE Int. Conf. Synthesis, Modeling, Anal. Simul. Methods and Appl. to Circuit Design, Sep. 2012, pp. 261-264.

[15] S. Sambandan, A. Kumar, K. Sakariya, and A. Nathan, "Analogue circuit building blocks with amorphous silicon thin film transistors," Electron. Lett., vol. 41, no. 6, pp. 314-315, 2005.

[16] P. Bahubalindruni, V. G. Tavares, P. Barquinha, C. Duarte, P. G. de Oliveira, R. Martins, and E. Fortunato, "Transparent current mirrors with a-GIZO TFTs: Neural modeling, simulation and fabrication," $J$. Display Technol., vol. 9, no. 12, pp. 1001-1006, Dec. 2013.

[17] P. Calzolari, G. Masetti, and M. Severi, "Integrated n.m.o.s. operational amplifier for c.c.d. transversal filters," Electron. Lett., vol. 15, no. 1, pp. 29-31, 1979.

[18] S. Sambandan, "High-gain amplifiers with amorphous-silicon thin-film transistors," IEEE Electron Device Lett., vol. 29, no. 8, pp. 882-884, Aug. 2008.

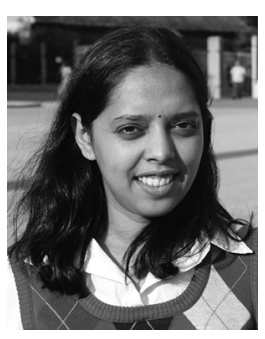

Pydi Ganga Bahubalindruni received the B.Tech. degree in electronics and communications and M.Tech. degree in electronic instrumentation from JNTU and NIT Warangal in India, respectively. Currently she is a Ph.D. student at FEUP/INESC in Department of Electrical and Computer Engineering.

Prior to beginning her doctoral studies, she was a Technical Member in Flowgic India, Chennai from August 2007 to February 2009. Her research interests include reconfigurable ASIC design, modeling of semiconductors with neural networks and design of analog/mixed signal circuits with CMOS and emerging technologies.

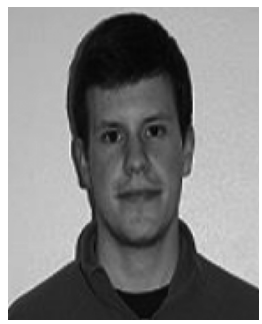

Bruno Silva was born in Porto, Portugal, on May 30 1990. He received the M.S. degree from Electrotechnical and Computer Engineering at FEUP in 2013. His these focused on the development of high-gain opamps with a-GIZO TFTs.

After finishing the course, he worked as a Research Fellow with INESC Porto for half a year and since March 2014. He is currently an Analog Designer with Synopsys, developing analog IP in cutting-edge deep submicron technologies.

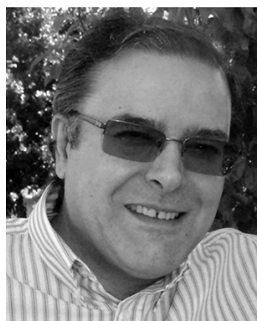

Vítor Grade Tavares received the Licenciatura and M.S. degrees in electrical engineering from the University of Aveiro, Portugal, in 1991 and 1994, and the $\mathrm{Ph} . D$. degree in electrical engineering from the Computational NeuroEngineering Laboratory, University of Florida, Gainesville, FL, USA, in 2001

In 1999, he joined the University of Porto, Portugal, as an Invited Assistant, where he has been an Assistant Professor since 2002. He is also a Senior Researcher at the INESC Porto. His research interests include low power, mixed signal and neuromorphic integrated-chip design and biomimetic computing, CMOS RF integrated circuit design and transparent electronics. In 2010 he was a Visiting Professor at Carnegie Mellon University, Pittsburgh, PA, USA.

Prof. Grade Tavares was a co-founder and chairman of the Portuguese IEEE Education Society Chapter.

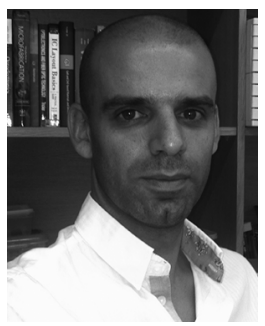

Pedro Barquinha received the Ph.D. degree in nanotechnologies and nanosciences from Universidade Nova de Lisboa in 2010.

$\mathrm{He}$ has been involved in transparent electronics from 2004, and his work on this area contributed to take performance and integration of this technology to levels of great interest to the display industry. He is coauthor of more than 80 peer-reviewed papers, with more than 3000 citations (h-index $=28$, as September 2014). He coauthored one book and two book chapters on this area as well. He won several national scientific prizes and gave more than 20 invited lectures including 2 key-notes in international scientific conferences and workshops. He is currently an Assistant Professor at FCT-UNL and his research interests are: design, fabrication, and characterization of electronic circuits using multicomponent oxides processed by physical and solution processing routes at low temperature for flexible and transparent displays; nanofabrication and nanoanalysis tools for oxide materials and devices. 


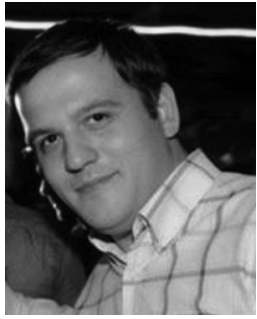

systems and FPGAs.

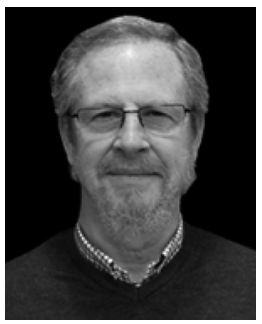

Nuno Cardoso was born in Porto, Portugal, in 1986. He received the Ms.C. degree in electrical and computer engineering from the University of Porto, Portugal (FEUP), in 2012. His thesis was entitled "Development of an ARGOS beacon for the VORSat Cubesat."

After that, he joined INESC-TEC as a Research Fellow, where he engaged into the SELF-PVP project until June 2014. Currently he is working at Synopsys as a Physical Design Engineer. His main interests are microelectronic circuit design, digital

Pedro Guedes de Oliveira is Professor of Electronics with the Faculty of Engineering of the University of Porto (FEUP), and his research interests are in the area of electronic circuits and microelectronics, signal processing and biomedical applications. He was formerly President of INESC Porto and is, at present, head of Electrical and Computer Engineering Department of FEUP.

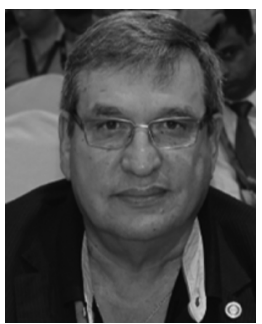

Rodrigo Martins is a Full Professor of Faculty of Sciences and Technology of New University of Lisbon, Portugal, in the field of Advanced Functional Materials and Microelectronics, where he is also $\mathrm{He}$ is Head of Materials Science Department, member of the Portuguese Academy of Engineering and of the Portuguese Order of Engineers. Immediate Past President of the European Materials Research Society (E-MRS) and running president of the senate of E-MRS. Founder and director of the Centre of Excellence in Microelectronics and Optoelectronics
Processes of Uninova; leader of the Materials, Optoelectronics and Nanotechnologies group of I3N/CENIMAT. Expert in the field of transparent electronics and on the paper electronics Member of the: advisory board of Horizon 2020 (Advanced Materials, Nanotechnologies, Biotechnologies and Manufacturing); International Advisory Board of Strategic Initiative Materials, a Flanders industry initiative; International Advisory Quality Assurance Board of the ERASMUS MUNDUS MASTER COURSE FAME (Functionalized Advanced Materials and Engineering); Steering Committee of EuMat; Alliance for Materials; Joint Innovation Centre for Advanced Material, Sino-Portuguese; External Advisory board and steering committee of EU KNIGHTS. Coordinator of the Portuguese Ph.D. AdvaMTech program; member of the GTA group of the Portuguese Foundation for Science and Technology;

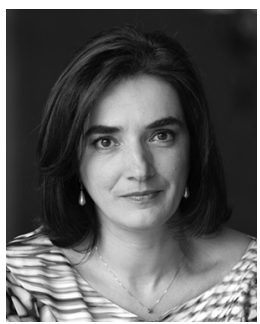

Elvira Fortunato is a Full Professor of Materials Science at the Faculty of Sciences and Technology of Universidade Nova de Lisboa, since 2012, specialized in Microelectronics and Nanotechnologies. Presently she is Director of the $\mathrm{i} 3 \mathrm{~N}$ and CENIMAT and member of the Advisory group of the EU Horizon 2020 program connected to DG CONNECT. She pioneered European research on transparent electronics, namely thin-film transistors based on oxide semiconductors, demonstrating that oxide materials may be used as true semiconductors as well as co-inventor of the paper transistor and electrochromic transistor. She is also Director of the Ph.D. program in Micro and Nanotechnologies Engineering; Associate editor of Pysica Status Solidi Rapid Research Letters, Wiley; Co-Editor of Europhysics Letters; Member of the Advisory Editorial Board of Applied Surface Science; Member of the National Scientific and Technological Council and Member of Academia de Engenharia, Portugal. She filled 68 international patents and got more than 15 international prizes for her work. In 2008 she wins an Advanced Grant from ERC for the project Invisible (first edition and she got the first position), which was also considered by ERC as a success story. 